\title{
Avaliação da Adesão ao Tratamento com Antidepressivos em Pacientes de uma Farmácia Pública no Interior do Ceará
}

\author{
José Marcio Andrade ${ }^{1}$, Francisca Andreza Fernandes de Souza ${ }^{2}$, Joeldo Ferreira Duarte ${ }^{3}$, \\ Pedro Ivo Palácio Leite ${ }^{4}$, Poliana Moreira de Medeiros Carvalho
}

\begin{abstract}
Resumo: A prevalência estimada de transtornos mentais é cerca de $12 \%$ na população mundial, sendo a maior parcela medicada na Atenção Primária à Saúde, o sucesso da terapia antidepressiva depende do profissional de saúde que tem papel importante nas questões relativas à adesão do tratamento. O Sistema Único de Saúde foi sem dúvida o maior movimento de inclusão social que a população já viu na história do Brasil, em termos constitucionais, como afirmação política de compromisso do estado brasileiro para os direitos da cidadania. $\mathrm{O}$ objeto de estudo foi avaliar a prevalência e adesão da terapia antidepressiva em pacientes atendidos em uma farmácia municipal da cidade de Juazeiro do Norte/CE no período de agosto de 2016. Desenvolveu-se o estudo, de caráter quantitativo, transversal e descritivo, em um Sistema de Atendimento Médico Especializado (SAME) no município de Juazeiro do Norte. Coletaram-se os dados por meio de um questionário socioeconômico e farmacoepidemiolôgico. Consideraram-se aderentes os pacientes que compareceram à farmácia para recebimento dos antidepressivos durante todo o mês da pesquisa. Participaram da pesquisa 50 pacientes dentre os quais, $88 \%$ eram do gênero feminino e $22 \%$ do gênero masculino, a faixa etária média foi de $34 \%$ entre $49-58$ anos. E que $58 \%$ dos pacientes tinha escolaridade com $1^{\circ}$ grau incompleto, 58\% disseram não trabalhar e estado civil prevaleceu com $40 \%$ para casados. Observou-se a maior prevalência do uso de amitriptilina e fluoxetina para cada com $48 \%$, e $50 \%$ responderam usar o medicamento para tratamento da depressão. O estudo obteve $96 \%$ de assiduidade satisfatória quanto ao grau de adesão. O farmacêutico é um profissional com amplo conhecimento farmacológico capacitado para manejar o atual arsenal terapêutico e informar sobre o uso e os efeitos dos fármacos. Assim, esta prática melhoraria indiscutivelmente a saúde e a qualidade de vida do paciente.
\end{abstract}

Palavras - chave: Antidepressivos. Adesão de Psicofármacos. Sistema Único de Saúde.

\section{Evalution of the Adhesion of the Treatment with Antidepressives in Patients of a Public Pharmacy in the Interior of Ceará}

\begin{abstract}
The estimated prevalence of mental disorders is about $12 \%$ in the world population, the largest portion being medicated in Primary Health Care, the success of antidepressive therapy depends on the health professional who plays an important role in issues related to treatment adherence. The Unified Health System was undoubtedly the largest movement of social inclusion that the population has ever seen in the history of Brazil, in constitutional terms, as a political affirmation of the Brazilian state's commitment to the rights of citizenship. The objective of this study was to evaluate the prevalence and adherence of antidepressive therapy in patients treated at a municipal pharmacy in the city of Juazeiro do Norte / CE during the period of August 2016. The quantitative, cross-sectional
\end{abstract}

\footnotetext{
${ }^{1}$ Curso de Farmácia pela Faculdade de Medicina Estácio em Juazeiro do Norte - FMJ. Contato: jmarcio92@hotmail.com;

${ }^{2}$ Graduação em farmácia pela Faculdade de Medicina Estácio em Juazeiro do Norte, Brasil. Auxiliar de Saúde Bucal da Prefeitura Municipal de Juazeiro do Norte , Brasil.

${ }^{3}$ Graduando em farmácia pela Universidade Estácio - Juazeiro do Norte.

${ }^{4}$ Graduado em Ciências Farmacêuticas pela Faculdade de Medicina Estácio de Juazeiro do Norte.

${ }^{5}$ Doutoranda em Ciências da Saúde pela Faculdade de Medicina do ABC Paulista, Cursando Especialização em Gestão e Docência Ensino Superior na Faculdade Estácio - FMJ, Bacharel em Farmácia, farmacêutica industrial e bioquímica clínica, especialista em Manipulação Magistral pela Universidade de Fortaleza e mestra em Bioprospecção Molecular pela Universidade Regional do Cariri - URCA. Professora da Faculdade de Juazeiro do Norte- FJN, Coordenadora da especialização em Atenção Farmacêutica - pela FJN. Professora da Faculdade de Medicina Estácio FMJ, do curso de Farmácia. Cursando especialização em Docência do Ensino Superior na Faculdade de Medicina Estácio - FMJ.
} 
study and descriptive, in a Specialized Medical Care System (SAME) in the city of Juazeiro do Norte. The data were collected through a socioeconomic and pharmacoepidemiological questionnaire. Patients who attended the pharmacy to receive antidepressants during the entire month of the study were considered as adherents. Results: Fifty patients participated in the study, of which $88 \%$ were female and $22 \%$ were male. Mean age was $34 \%$ between 49-58 years. And that 58\% of patients had incomplete elementary school education, $58 \%$ said they did not work and married status prevailed with $40 \%$. The highest prevalence of use of amitriptyline and fluoxetine for each was observed with $48 \%$, and $50 \%$ responded using the drug for the treatment of depression. The study obtained $96 \%$ of satisfactory attendance regarding the degree of adhesion. The pharmacist is a professional with extensive pharmacological knowledge able to handle the current therapeutic arsenal and report on the use and effects of the drugs. Thus, this practice would undoubtedly improve the patient's health and quality of life.

Keywords: Antidepressants. Adhesion of Psychopharmaceuticals. Unified Health System.

\section{Introdução}

Segundo a Organização Mundial de Saúde (OMS), no ano 2000, a depressão foi a principal causa de incapacidade no mundo avaliada pelo índice de anos vividos com incapacidade e a quarta causa de perda de dias de produção no trabalho. Considerando a frequência elevada a OMS projeta que a depressão será o segundo maior problema de saúde pública em 2020 (SILVIA, 2011). A prevalência estimada de transtornos mentais é cerca de 12\% na população mundial, sendo a maior parcela medicada na Atenção Primária à Saúde (APS) (ROCHA et al., 2013). Uma revisão sistemática elaborada por Silva et al. (2014), destaca que a prevalência de sintomas depressivos pela população pesquisada nos estudos foi de $14 \%$ (mínimo de 13 e máximo de 16, Intervalo de Confiança 95\%) Berghöfer et al. (2011), sublinha que a depressão é um dos principais motivos de consulta médica nos serviços de APS, sendo o transtorno mental mais prevalente.

Estima-se que $23 \%$ da população brasileira utilizam $60 \%$ da produção nacional de medicamentos, e que o uso destes vem crescendo com o aumento da faixa etária, possivelmente, se deve à maior prevalência de morbidades com o avanço da idade (GARCIAS et al., 2008).

A terapia com antidepressivos (AD) é considerada como tratamento de escolha nas manifestações agudas da depressão moderada e grave, seja para melhoria sintomática, seja para tratamento completo. Também podem ser utilizada no tratamento de outros problemas de saúde, como dores crônicas, transtorno obsessivo compulsivo (TOC), síndrome do pânico e transtornos da ansiedade (LOYOLA FILHO et al., 2014). O medicamento, que faz parte de todo complexo médico-industrial, demonstra influência na percepção da saúde e doença 
passando a ser visto como a solução "mágica" para os problemas da população, assumindo o conceito de bem de consumo dos medicamentos em detrimento ao de bem social. Apesar disso, não é apresentada apenas como uma substância química isolada, mas vêm acompanhadas por um conjunto de publicidade, informações, brindes, estudos, entre outras coisas, que busca interferir na forma de pensar do consumidor (VIEIRA, 2007).

A depressão é uma doença caracterizada por uma síndrome envolvendo diversos mecanismos patogênicos e etiológicos, multifatoriais, através de uma menor liberação de monoaminas endógenas como a serotonina (5-HT), a noraepinefrina (NE) e a dopamina (DA), nas sinapses de neurônios cerebrais (KATZUNG; MASTERS; TREVOR, 2014). Diante disso, a ausência de serotonina pode explicar os problemas com ansiedade, obsessões e compulsões, já a ausência de noraepinefrina está relacionada com a perda de energia, atenção e interesse pela vida, e a dopamina, quando diminuída, liga-se à redução de atenção, motivação e prazer (RANG et al., 2007).

O uso inadequado de medicamentos pela população é a principal consequência do consumo exagerado, contribuindo para o surgimento de vários eventos adversos, aumentando o risco de mortalidade e morbidade, além disso, elevando os custos com a saúde (VIEIRA, 2007). Neste sentido, desde a Declaração de Tóquio em 1993, a OMS vem tentando reforçar a grande importância da incorporação do profissional farmacêutico à equipe de saúde, visto que esse seria o profissional de melhor capacitação para o manejo destas ações (MARIN et al., 2003).

Carvalho, Merchan-Hamann e Matsushita (2007), observaram em outros estudos sobre prevalência de adesão ao tratamento, que alguns fatores foram determinantes do nível de adesão como, por exemplo, a idade, escolaridade, situação de emprego, rendas pessoal e familiar, uso de substâncias ilícitas, estrutura familiar e a presença de efeitos colaterais relacionados ao tratamento. Também foi possível verificar que alguns indivíduos não eram aderentes por não saber a forma correta de tomar a medicação ou o número de comprimidos que deveriam ingerir.

O farmacêutico desempenha um papel ideal no programa de adesão. Fatores que influenciam a adesão, como a toxicidade previsível, o esquema terapêutico, a posologia e preferências em relação ao esquema de medicamentos devem ser esclarecidos com detalhes, com conversas individuais de cada paciente com o profissional farmacêutico (BRASIL, 2010).

Investigar o perfil do consumo de antidepressivos em uma farmácia pública é necessário planejar intervenções na comunidade e com os prescritores, promovendo o uso racional dos 
medicamentos, incluindo a seleção dos mesmos na Relação Nacional de Medicamentos Essenciais (RENAME). Tendo em vista esta panorama, o objeto desta é verificar a prevalência e a adesão do consumo de antidepressivos em usuários adultos de uma farmácia pública no município de Juazeiro do Norte (CE).

\section{Metodologia}

Trata-se de estudo descritivo, transversal, na qual a abordagem é do tipo quantitativo. Esta pesquisa foi executada através de dois questionários que correlacionam o comportamento de adesão do tratamento com dados e informações necessárias pelo próprio paciente.

Está pesquisa foi realizada na farmácia do Sistema de Atendimento Médico Especializado (SAME), no município de Juazeiro do Norte-CE, onde, de acordo com (GERHARDT; SILVEIRA, 2009), esse tipo de pesquisa de campo é caracterizada pela realização de coleta de dados juntamente com pessoas, com o intuito de vários tipos de pesquisa.

50 pacientes adultos com faixa etária entre 18 e 65 anos de ambos os sexos foram abordados no ano de 2018 na farmácia do Serviço Especializado da atenção básica. Foram incluídos na pesquisa pacientes presentes no estabelecimento em busca de sua medicação dispensada pela farmácia, e foram exclusos da pesquisa os pacientes que poderiam apresentar condições de agressividade, surto e gestantes.

O primeiro passo foi solicitar autorização junta secretaria de saúde local da cidade para realização da pesquisa no SAME. Após a aceitação do projeto, a pesquisa foi apresentada aos participantes por meio da apresentação do TCLE (Termo de Consentimento Livre e Esclarecido), que foi devidamente assinado, lido e confirmando a participação no estudo de cada paciente.

Os dados obtidos dos questionários foram digitados no software Microsoft Office Excel 2010, e convertido banco de dados no programa Epi. Info 7.0, onde foi feita análise estatística descritiva, com produção de tabelas e gráficos de distribuição de frequências simples e de contingência. Foram graduadas e calculadas de acordo com o questionário estabelecido por (REMOR; MILNER-MOSKOVICS; PREUSSLER, 2007), onde apresentaram uma pontuação para cada resposta de acordo com a pergunta, e ao final da análise do somatório das respostas o paciente compôs uma nota a qual foi comparada em uma tabela que classificou como: grau 
satisfatório de 65 a 89 pontos, moderado de 41 a 64 pontos e não satisfatório de 17 a 40 pontos. A análise da pontuação total obtida foi feita pela soma de todos os itens do questionário farmacoepidemiológico, onde variou de 17 pontos (valor mínimo) e 89 pontos (valor máximo). Quanto maior a pontuação, maior adesão ao tratamento.

A pesquisa foi submetida ao comitê de ética em pesquisa da FJN - Faculdade de Juazeiro do Norte-CE, de acordo com a portaria 466/12, protocolo CAAE: 57516716.7.0000.5624 onde os dados só foram colhidos após aprovação do projeto. Os participantes foram expostos a riscos, porém estes foram minimizados, pois passaram por questionário individual de forma oral, explicado e relatado ao paciente as perguntas, em uma sala separada, garantindo total sigilo das informações fornecidas e anonimato da sua identidade.

\section{Resultados e Discussão}

A população estudada foi de 50 pacientes sendo sua maioria mulheres, com $44(88 \%)$ participantes do gênero feminino e $6(12 \%)$ do gênero masculino, apresentando para ambos os sexos 17 (34\%) com idade entre 49 e 58 anos. É possível observar a maior prevalência do uso de antidepressivos nas faixas etárias superiores a 30 anos, fato corroborado com Almeida, Coutinho e Pepe (1994) de que o maior consumo de psicofármacos associou-se significativamente com o aumento da idade. No que diz respeito à prevalência de mulheres com distúrbios mentais o sexo é um fator que parece ter influência no surgimento do mesmo. Segundo a Organização Mundial de Saúde (2011), as mulheres são mais suscetíveis a adquirirem algum tipo de transtorno mental. Nos países de baixa renda, as mulheres que vivem nas residências mais pobres relatam mais problemas de saúde mental do que aquelas que vivem nas de classe alta (o que também se pôde observar no nível de escolaridade apresentada pela amostra dos questionários desse estudo).

Com relação à escolaridade, $29(58 \%)$ dos participantes afirmaram ter o ensino fundamental incompleto, $14(28 \%)$ responderam ter concluído o ensino médio, 4 (8\%) concluíram o ensino fundamental, e 1 (2\%) tem ensino médio incompleto. Apesar de que 29 (58\%) da amostra encontram-se em condições desvantajosas para entender os riscos / efeitos colaterais dos medicamentos e assimilar as informações oferecidas pelos profissionais de saúde, o estudo apresentou um grau satisfatório na adesão dos medicamentos. Em relação à situação 
conjugal dos participantes os dados revelaram que os maiores números de usuários de antidepressivos eram casados, ou seja, 20 (40\%), visto que 17 (34\%) eram solteiros, 6 (12\%) eram divorciados, $4(8 \%)$ eram viúvos, 4 (8\%) eram separados e 1 (2\%) união estável. Diante disso, estudos mostram que indivíduos com companheiros e solteiros consumiram significativamente mais psicofármacos que os viúvos e divorciados (RODRIGUES; FACCHINI; LIMA, 2006).

Os dados relativos à ocupação revelam que, 29 (58\%) dos usuários de antidepressivos não trabalham, e 21 (42\%) disseram trabalhar. No estudo de Hildebrandt et al. (2009), prevaleceu a profissão de pessoas que desempenham suas atividades domiciliares seguidas pelos aposentados. Em relação aos aposentados demonstram que estão em uma fase da vida, em que em virtude das condições, apresentam altos índices de modificações orgânicas, psíquicas o que, às vezes, gera ansiedade, angústia, medo e sofrimento emocional.

Tabela 1 - Distribuição da Amostra de Pacientes Segundo Dados Sociodemográficos, Antidepressivos e Principais Doenças.

\begin{tabular}{llcc}
\hline & & $N$ & $\%$ \\
\hline Idade & 18 a 28 anos & 1 & $2 \%$ \\
& 29 a 38 anos & 16 & $32 \%$ \\
& 39 a 48 anos & 10 & $20 \%$ \\
& 49 a 58 anos & 17 & $34 \%$ \\
& $>59$ anos & 6 & $12 \%$ \\
\hline Sexo & Feminino & 44 & $88 \%$ \\
& Masculino & 6 & $12 \%$ \\
\hline Escolaridade & Ensino Fundamental $\left(1^{\circ}\right.$ grau) Incompleto & 29 & $58 \%$ \\
& Ensino Fundamental $\left(1^{\circ}\right.$ grau) completo & 2 & $4 \%$ \\
& Ensino Médio $\left(2^{\circ}\right.$ grau) incompleto & 4 & $8 \%$ \\
& Ensino Médio $\left(2^{\circ}\right.$ grau) completo & 1 & $2 \%$ \\
& Superior Incompleto & 14 & $28 \%$ \\
& Superior Completo & 0 & 0 \\
& Pós Graduação & 0 & 0 \\
\hline \multirow{2}{*}{ Doençado Civil } & Solteiro (a) Casado (a) & 16 & $32 \%$ \\
& União Estável & 20 & $40 \%$ \\
& Viúvo (a) Divorciado (a) & 1 & $2 \%$ \\
& Separado (a) & 3 & $6 \%$ \\
& Ansiedade & 18 & $36 \%$ \\
& Depressão & 25 & $50 \%$ \\
& Enxaqueca & & \\
& & 1 & $2 \%$ \\
& Hérnia de Disco & 1 & $2 \%$ \\
& Insônia & 5 & $10 \%$ \\
\hline
\end{tabular}




\begin{tabular}{llll}
\hline Medicamentos & Amitriptilina & 24 & $48 \%$ \\
& Citalopram & 1 & $2 \%$ \\
& Fluoxetina & 24 & $48 \%$ \\
& Venlafaxina & 1 & $2 \%$ \\
\hline Trabalho & Sim & 21 & $42 \%$ \\
& Não & 29 & $58 \%$ \\
\hline
\end{tabular}

*N: 50 pacientes (total de pacientes).

Fonte: Pacientes atendidos na Farmácia do SAME.

Sobre o uso de antidepressivos no tratamento de doenças, 25 (50\%) tem depressão, 18 (36\%) responderam ter ansiedade, $1(2 \%)$ tem enxaqueca e $1(2 \%)$ com hérnia de disco. Vicente et al. (2015) refletindo o estudo de Rocha e Werlang pontuam que no Brasil há poucos estudos investigando a prevalência de uso de psicofármacos, tendo como seu padrão de uso na população, em especial na APS, observou-se que entre estes fármacos, a classe de fármacos mais prevalente foi a de antidepressivos, utilizados por $63,2 \%$ dos entrevistados, o que pode ser correlacionado com aumento do diagnóstico dos transtornos depressivos.

Os antidepressivos mais utilizados foram amitriptilina com 24 (48\%), fluoxetina 24 (48\%), 1 (2\%) citalopram e com 1 (2\%) venlafaxina. Em estudo de Vicente et al. (2015), Os inibidores seletivos da recaptação da serotonina (ISRSs) foram os antidepressivos mais amplamente utilizados, sexo feminino, idade avançada, auto-avaliação negativa da saúde, limitação funcional e histórico de depressão foram os indicadores sócio-demográficos e clínicos mais consistentes do uso de antidepressivos.

Tabela 2 - Respostas do Grau de a Adesão a Terapia de Antidepressivos do Questionário

\begin{tabular}{llc}
\hline & $N$ & $\%$ \\
\hline Satisfatório de 65 a 89 pontos. & 48 & $96 \%$ \\
\hline Moderado de 41 a 64 pontos. & 2 & $4 \%$ \\
\hline Não satisfatório de 17 a 40 pontos. & 0 & 0 \\
\hline
\end{tabular}

*N: 50 pacientes.

Fonte: Pacientes atendidos na Farmácia do SAME.

A adesão do paciente ao medicamentoso prescrito é um comportamento desejável na terapia de doenças crônicas, assim, na prática, pode-se observar que os pacientes e seus familiares apresentam várias dificuldades na manutenção do tratamento, como: presença de reações adversas, interação medicamentosa, esquemas com posologias inconvenientes e 
inadequados, não entendimento das instruções, educação em saúde inadequada para o paciente, deficiência dos serviços de saúde e relação médico/paciente deficiente, entre outras (CARDOSO et al., 2011).

O impacto do uso de medicamentos em uma sociedade tem várias facetas. Por um lado, os medicamentos podem aumentar a expectativa de vida, erradicar certas doenças, trazer benefícios sociais e econômicos, e por outro lado podem aumentar os custos da atenção à saúde se utilizados inadequadamente e ou levar à ocorrência de reações adversas/efeito colateral. (PFAFFENBACH; CARVALHO; MENDES, 2002).

Cabe ao profissional farmacêutico, orientar os usuários de psicofármacos durante a atenção farmacêutica, para que o paciente tenha consciência sobre os riscos/efeitos colaterais desses medicamentos. Principalmente quanto às possíveis complicações relacionadas ao uso prolongado do medicamento. É de extrema importância que o paciente tenha conhecimento dos efeitos colaterais que o uso dos psicofármacos pode levar, pois este conhecimento reduz a falha na adesão ao tratamento. Bem como, é necessário que os usuários saibam dos riscos que esse medicamento pode causar como dependência e tolerância.

\section{Considerações Finais}

Considera-se aqui a importância da inserção do farmacêutico na equipe interdisciplinar nas farmácias públicas, pois o mesmo seria complementar aos serviços assistenciais, promovendo uma orientação responsável do tratamento farmacológico objetivando a melhor adesão do tratamento, conhecimento dos medicamentos e da patologia e primordialmente a prevenção, identificação e resolução de problemas relacionados aos medicamentos (PRMs).

O farmacêutico é um profissional com amplo conhecimento farmacológico capacitado para manejar o atual arsenal terapêutico e informar sobre o uso e os efeitos dos fármacos. Assim, esta prática melhoraria indiscutivelmente a saúde e a qualidade de vida do paciente, isso demonstra a necessidade de um atendimento que seja capaz de esclarecer todas as dúvidas em relação ao uso dos medicamentos, para que não ocorram falhas devido à falta de informações. 


\section{Referências}

ALMEIDA, L. M.; Coutinho, E. S. F.; PEPE, V. L. E. Consumo de Psicofármacos em uma Região Administrativa do Rio de Janeiro: A Ilha do Governador. Cadernos de Saúde Pública, Rio de Janeiro, v. 10, n. 1, p.5-16 jan./mar.1994.

BRASIL. Ministério da Saúde. Protocolo em Assistência Farmacêutica em DST/HIV/AIDS: recomendações do Grupo de Trabalho de Assistência Farmacêutica. Brasília: Ministério da Saúde, 2010.

CARDOSO, L. et al. Grau de adesão e conhecimento sobre tratamento psicofarmacológico entre pacientes egressos de internação psiquiátrica. Rev. Latino-Am. Enfermagem, Ribeirão Preto, v. 19, n. 5, p. 1-9, set. 2011.

CARVALHO, Cláudio Viveiros de; Merchan-Hamann, EDGAR; MATSUSHITA, Raul. Determinantes da adesão ao tratamento antiretroviral em Brasília, DF: um estudo de caso controle. Rev. Soc. Bras. Med. Trop., Uberaba, v. 40, n. 5, Oct. 2007.

GARCIAS, Carla Maria Maia et al. Prevalência e fatores associados ao uso de antidepressivos em adultos de área urbana de Pelotas, Rio Grande do Sul, Brasil, em 2006 Prevalence of antidepressant use and associated factors among adults in Pelotas, Rio Grande. Cad. Saúde Pública, v. 24, n. 7, p. 1565-1571, 2008.

GERHARDT, T. E.; Silveira, D. T. Médotos de pesquisa. Porto Alegre: Editora da UFRGS, 2009.

HILDEBRANDT, L. M. et al. Prevalência no consume de psicotrópicos pela população assistida por um serviço municipal se saúde. 2009. Disponível em: <http://www.madres. org/asp/contenidoscielo.br>. Acesso em: 14 out. 2011.

KATZUNG, Bertram G.; Masters, Susan B.; Trevor, ANTHONY J. Farmacologia básica e clínica. 12. ed. São Paulo: AMGH, 2014.

LOYOLA FILHO, Antônio Ignácio et al. Tendências no uso de antidepressivos entre idosos mais velhos: Projeto Bambuí. Revista de Saúde Pública, v. 48, n. 6, p. 857-865, 2014.

MARIN, N. et al. Assistência farmacêutica para gerentes municipais de saúde. Rio de Janeiro: OPAS/OMS, 2003.

OMS - ORGANIZAÇÃO MUNDIAL DE SAÚDE. Mulheres e saúde: evidências de hoje agenda de amanhã. 2011. Disponível em: <http://www.progresso.com.br/caderno-a/cienciasaude/oms>. Acesso em: 13 de maio de 2016.

PFAFFENBACH, G.; Carvalho, O. M.; Mendes, G. B. Reações adversas a medicamentos como determinantes da admissão hospitalar. Revista Associação médica Brasileira, São Paulo, v. 48, n.3, p.237-241, 2002. 
RANG, H. P. et al. Farmacologia. 6. ed. Rio de Janeiro: Guanabara-Koogan, 2007.

REMOR, EDUARDO; Milner-Moskovics, JENNY; Preussler, GISELE. Adaptação brasileira do "Cuestionario para la Evalución de la Adhsión al tratamiento Antiretroviral". Rev. Saúde Pública, São Paulo, v. 41, n. 5, oct. 2007.

ROCHA, B.S. et al. Psicofármacos na Estratégia Saúde da Família: perfil de utilização, acesso e estratégias para a promoção do uso racional. Ciência e Saúde Coletiva, v. 18, n. 11, p. 32913300, 2013.

RODRIGUES, M. A.; Facchini, L. A.; Lima, M. S. Modificações nos padrões de consumo de psicofármacos em localidade do Sul do Brasil. Revista de Saúde Pública, São Paulo, v.40, n. 1, p.107-114, jan./fev. 2006.

SILVA, M. S. et al. A prevalência de morbidade depressão entre Brasileiros adultos: uma revisão sistemática e meta-análise. Revista Brasileira de Psiquiatria, v. 36, p. 262-270, 2014.

SILVIA, J. Depressão e trabalho: ruptura de laço social. Rev. bras. Saúde ocup., São Paulo, 36 (123): 84-92, 2011.

VICENTE et al. Uso de antidepressivos e fatores associados em idosos: o projeto bambuí. Ciência e Saúde Coletiva, v. 20, n. 12, p. 3797-3804, 2015.

VIEIRA, Fabiola Sulpino. Possibilidades de contribuição do farmacêutico para a promoção da saúde. Ciência e Saúde Coletiva, v. 12, n. 1, p. 213-220, 2007.

\section{Como citar este artigo (Formato ABNT):}

ANDRADE, José Marcio; SOUZA, Francisca Andreza Fernandes de; DUARTE, Joeldo Ferreira; LEITE, Pedro Ivo Palácio; CARVALHO, Poliana Moreira de Medeiros. Avaliação da Adesão ao Tratamento com Antidepressivos em Pacientes de uma Farmácia Pública no Interior do Ceará. Id on Line Rev.Mult. Psic., 2018, vol.12, n.42, p. 203-212. ISSN: 1981-1179.

Recebido: 23/08/2018;

Aceito: 28/08/2018 